\title{
Robotic Process Automation: Lessons Learned from Case Studies
}

\author{
Cristina-Claudia OSMAN \\ Business Informatics Research Centre, Romania \\ cristina.osman@econ.ubbcluj.ro
}

\begin{abstract}
Nowadays, there is a trend in automating repetitive tasks in order to reduce human errors or costs. Digitization asks for new strategies in business processes. Robotic Process Automation (RPA) includes a set of emerging technologies that promises the automation of business processes by using software robots that are trained based on human tasks. Companies need to constantly monitor their own business processes in order to identify and optimize processes suitable for automation. In 2018, Forrester identified UiPath, Automation Anywhere, and Blue Prism as being the leaders providing RPA solutions by using 30-criteria evaluation. This paper examines ten case studies of companies integrating RPA and presents a series of lessons provided by practice. Not all processes are suitable for automation, all case studies identifying five fundamental criteria to be considered.
\end{abstract}

Keywords: Robotic Process Automation, RPA, BPMS, Process Mining, Case Study

\section{1} Introduction

The emerging of new digital tools supports organizations to improve their business processes by increasing efficiency and agility, or reducing errors and costs. Robotic Process Automation (RPA) tools are designed to perform manual and repetitive tasks of human employees using trained robots. This is different than other traditional software because the robots communicate with other Information Systems by means of front-end. RPA helps companies to reduce employees' workload and errors, but also to save costs.

Nowadays, the development of IT solutions is divided into two main approaches: lightweight IT and heavyweight IT [1]. Lightweight IT refers to mobile apps, sensors and Internet-of Things, while heavyweight IT is represented by traditional systems and their integration. RPA is seen as being a lightweight IT-enabled innovation, while traditional back-end system automation as heavyweight IT-enabled innovation [2],[3]. Heavyweight IT relies on Software Engineering, while lightweight IT on business innovation [1].

Though, process automation is not a new field. In mid-nineties, financial institutes started implementing automated electronic payment processes, so-called "StraightThrough Processing" [4]. The aim was to eliminate human beings' intervention within the entire payment process.

Process automation is not a trivial task. The literature shows nine criteria to be considered in process automation: a) high volume of transactions, b) limited exception handling, c) manual IT processes prone to errors or reworks, d) limited human intervention, e) stable environment, f) frequent access to multiple systems, g) high value of transactions, h) ease of decomposition into clear IT processes, and i) clear understanding of current manual costs [5].

There are studies which demonstrate that initial RPA projects fail in proportion of $30 \%$ to $50 \%$ [6]. This paper's aim is to provide insights of successful RPA implementation projects. To investigate the receipt of successful RPA implementation and what factors are critical, ten case studies are presented and analyzed. Seven of them represent academic research, while three of them are conducted by the leaders providing RPA solutions according to [7].

The remainder of this paper is divided into seven sections. After this introduction, second section provides a short history of Robotic Process Automation (RPA) and how it affects nowadays organizations. Next, a comparison between Business Process Management Systems (BPMSs) and RPA follows. Fourth Section presents Process Mining field as an assistant of RPA. Following section represents the 
core of this paper: the depiction of ten case studies about RPA implementation. Both academic and industrial case studies are introduced. After the case study section a discussion section follows and, finally, conclusions summarize common findings based on case studies.

\section{Robotic Process Automation (RPA)}

The literature provides several definition for Robotic Process Automation (RPA). The term was first used in 2012 by Blue Prism's Marketing Director Patrick Geary [8]. Although, European Patent Office (EPO) recognizes Cyrille Bataller and Adrien Jacquot as inventors of RPA [10]. They define RPA as being " $a$ technology that enables to automate the execution of repetitive and manually intensive activities." Gartner proposes another definition of RPA: "RPA is a productivity tool (sold as licensed software) that allows a user to configure one or more scripts (which some vendors refer to as "bots") to activate specific keystrokes in an automated fashion." Sutherland considers that Robotic Automation is the "application of specific technology and methodologies to use a computer or $<<$ virtualized FTE or robot $>>$ rather than a person to manipulate existing application software (e.g., ERPs, claims applications, databases, learning management systems) in the same way that a person today processes a transaction or completes a process."

All definitions emphasize the main purpose of RPA: the automation of repetitive tasks by using computers or robots. Once the tasks are automated, costs reduce, problems with management and miscommunication are eliminated [11],[12], execution time of tasks reduces [13], productivity and accuracy increase [14], and human errors are eliminated or mitigated [11], [12]. Moreover, RPA solutions can be implemented and used by non-IT employees [2]. Although, more complex situations ask for programmers [15].

Studies show that RPA implementation reduce FTE costs by 50 percent, while Back Office failure customer calls also decrease by 50 percent [2]. Furthermore, robots cost only
$10 \%$ of a FTE in an onshore location like UK [16].

Not all processes are suitable for automation. The most appropriate processes are those having rule-based tasks and do not require human judgment. In contrast to other methodologies like BPM, RPA operates only at graphical user interface (GUI) level as it simulates human behavior [11]. Any change of the application or of the process demand the reconfiguration of the robot.

Being an IT technology, RPA can only be applied on electronic data and all data must support the same format in order to be read and manipulated by bots. That is why the quality of data is a critical aspect. Any change of technology in the daily work of an employee is seen with skepticism, but there are studies showing that employees accept tasks automation as their employers engage them in more interesting and cognitive tasks [17].

\section{Business Process Management Systems (BPMSs) vs RPA}

BPMSs are "generic software systems that are driven by explicit process designs to enact and manage operational business processes" [19]. Paul Harmon sees RPA as a scaled down version of BPMSs [15]. Although RPA and BPMS present similarities as both of them play a significant role in digital transformation of enterprise, there are slight differences between them.

The major aim of RPA is to automate repetitive tasks, while BPMS focus not only on automation, but also on process improvement and decision support. On the other hand, RPA is considered lightweight IT, while BPMS heavyweight IT. Therefore, RPA acts at GUI level of an existing application, while BPMS development require programming skills. That is the reason why RPA can be implemented faster than a BPMS. Platforms like Google or Amazon may connect heavyweight and lightweight IT [1].

Also, from costs point of view, RPA is lowpriced than implementing a BPMS [2],[20]. Furthermore, RPA requires less resource and time investment [2].

Business Process Automation (BPA) module 
within a BPMS is more suitable for repetitive tasks, tasks that are duplicated in other processes, inefficient or outdated workflow processes, or new businesses or IT initiatives [21]. Moreover, automation is an opportunity to simplify processes and get rid of redundant or non-value adding tasks [21]. Same assumptions can be applied on RPA implementation. Another difference between BPMSs and RPA is that BPMSs need a database and a data model to store data, while RPA does not store any transactional data [22].

RPA should be seen as a complement of BPMSs or other type of system or IT technology, not as its rival as automating existing processes requires a structured approach to process analysis which is a critical aspect for RPA.

\section{Process Mining}

Process Mining is a domain including a series of methods and techniques that transform event $\log _{s}$ generated by Information Systems into visual representations like Petri Nets, BPMN diagrams, Social Networks, etc. [23]. Therein, besides process discovery, it also allows the analysis and comparison of To-Be processes to As-Is processes by using conformance checking algorithms. Process improvement using information stored in event logs represents the third Process Mining type: enhancement.

Process analysis using Process Mining techniques helps users to identify deviations and bottlenecks of processes and their causes. Also, Process Mining represents the one of the main enablers of digital transformation through process improvement in terms of efficiency, speed, agility and compliance [24]. Two case study combining Process Mining with RPA are described in Section 5. The use of Process Mining together with RPA accelerates the implementation of RPA [24].

\section{RPA Case studies}

The cases studies introduced in this section consists of two main categories: case studies presented by academic researchers and case studies presented by industry (top 3 leaders providing RPA solutions).

\subsection{RPA Case studies from academic envi- ronment}

This section introduces six case studies conducted by academia, where companies integrate RPA in order to support operational processes from different domains like telecommunications, insurance, outsourcing, and public administration. All studies are recent, being published starting with 2015 until 2019.

\subsubsection{Telecommunications processes}

First case study presents the findings of implementing RPA and CI within the second mobile telecommunications provider from UK, Telefónica O2, [18]. Although some companies are doubtful to embrace new technologies, this study emphasizes the benefits brought by the integration of RPA and CI techniques. This investigation was conducted by researchers from London School of Economics and Political Science (LSE). In order to transform low-performance back-offices into high-performance offices, the authors define six levers: centralize, standardize, optimize, relocate low cost area, technology-enable, and automation.

Telefónica $\mathrm{O} 2$ has automated $35 \%$ of their transactions by using Blue Prism ${ }^{1}$ software. Accordingly, the company automated 15 fundamental processes by using 160 robots that perform between 400000 and 500000 transactions monthly.

The entire transformation process began even since 2004, when the company started the outsourcing to India. Six years later, Telefónica O2 used RPA within two processes: SIM swaps and application to a pre-calculated credit. The pilot project took two weeks. The IT team of Telefónica $\mathrm{O} 2$ also proposed the automation of the process reminded earlier by using BPMSs. Although, the implementation took three weeks on terms of costs a greater payback is obtained when BPMSs are used (10 months versus 3 years). During the entire

\footnotetext{
${ }^{1}$ https://www.blueprism.com/
} 
training program, three employees of Telefónica $\mathrm{O} 2$ were familiarized with the RPA implementation. In 2015, 15 fundamental processes have been automated.

One of the conclusions of the study places the implementation of RPA together with other technologies like BPMS. Moreover, RPA is more suitable for tasks that are executed frequently and are based on rules, but it also takes some risks.

\subsubsection{Insurance processes}

XChanging $^{2}$, now part of DXC Technology ${ }^{3}$, is a company that provides digital solutions for business processes. DXC Technology resulted from the merging of CSC with Hewlett Packard Enterprise in 2017. Previously, in 2016, CSC completed the acquisition of XChanging.

Although, XChanging is now DXC Technology, the case study presented in this section was conducted during the period XChanging did not belong to DXC Technology. Therefore, we will refer to XChanging from now on. The case study describes the automation of 14 core insurance processes consisting of 120000 cases involving 27 robots [25]. The implementation of RPA led to costs savings of approximatively $30 \%$ per process. The RPA process implemented by XChanging started on 2013 and finished 2 years later.

Likewise Telefónica O2, XChanging also used Blue Prism software and focused on back office processes. The processes chosen for automation are those having a high volume and low complexity.

The conclusions of this study assume that RPA implementation projects not only needs the involvement of a RPA experts, but also a project coordinator. Besides, a business strategy that focuses on digitization by embracing innovation and technology helps on the adoption of RPA. Based on Six Sigma philosophy, the automation of a process starts after the process is standardized and stabilized.

\subsubsection{Outsourcing}

The case studies mentioned in this section belong to outsourcing companies. The first case study talks about financial processes, while the second describes the generation of a payment receipt process using RPA.

\subsubsection{Financial processes}

OpusCapita is a Finnish company providing solutions for the digitization process [11]. The company has implemented electronic invoicing since 1990s, while in 2000s their focus was on process automation.

The RPA implementation process starts with a workshop that takes 2 hours. The second step lasts approximatively one day and consists of process evaluation by examining the employees while executing tasks. Then the business case is presented to clients by highlighting the productivity improvement and costs reduction. If the business case is approved, the configuration of the robot starts and it is delivered to the client. The way of how OpusCapita used their RPA capabilities is depicted in [26]. RPA implementation was supported by UiPath.

OpusCapita believes that a small company having a labour-intense routine is more suitable to embrace RPA than a large multi-national company having few automatable tasks.

\subsubsection{Generation of a payment receipt}

The case study of Aguirre and Rodriquez [12] presents a business situation where both back and front office employees are involved into RPA implementation. Firstly, they depicted the As-Is process for generation of a payment receipt, where back and front office agents are involved.

The process starts when a customer gets in touch with an employee from the call centre and requests a payment receipt. After the request is received, the front office agent creates a new case in CRMS. Then, the case is opened by a back-office agent and the payment receipt is generated and sent to the customer via email. Last activity is also performed by a back-office agent which closes the case.

\footnotetext{
${ }^{3}$ https://www.dxc.technology/
} 
The To-Be process replaces the back-office employees with robots. The process starts similarly, but after the case is created, a robot automatically takes the information from CRMS and generates the payment receipt which is sent to the customer and the case closes.

The case study also depicts the comparative results of As-Is and To-Be processes. The results show the doubling of executed cases of To-Be process, relative to As-Is process. Although, the mean case duration is almost the same.

\subsubsection{Document analysis in Public Admin- istration}

Processes' digitalization has brought changes also in Public Administration sector. RPA techniques changes the entire way of how clerks from Public Administration perform their daily work. The research conducted by [27] identifies three types of RPA: programmable RPA, self-learning RPA, and cognitive RPA. Furthermore, a case study demonstrating a new cognitive RPA approach using a tax assessment scenario is described.

The case study emphasizes the role of RPA in the digitization process from Public Administration. After the digitization process is finished, the authors do not see RPA as powerful as it is in present, but RPA can also be used in automating repetitive tasks from other processes. In their work to automate data extraction from documents, deep convolutional neural networks are used.

The information from paper-based documents has to be digitized, but this process is challenging and classical OCR methods are not efficient all the time. Houy et al. propose a prototype of cognitive RPA based on object detection network. The position coordinates of each identified object within an image is reported. The cognitive RPA consists of two parts: a) document classification model and b) object detection model.

A flask web application is used in order to automate the reading of a document. After each object is detected, the data is extracted and then automatically stored into the legacy system.

The results of the case study demonstrate the efficiency of using RPA in Public Administration domain.

\subsubsection{Purchase-to-Pay process}

This section introduces two distinctive case studies where RPA is used together with Process Mining. Process Mining accelerates the implementation of RPA by restructuring processes before RPA implementation by decreasing time and cost of the robots' training [24].

The authors propose an approach consisting of three steps: assess, develop, and sustain. First phase includes the identification of the processes to be automated. Second phase consists of the pilot project where robots are trained with the existing workflow. Then, by applying Process Mining techniques on the generated process instances, the most suitable processes are chosen. The final step consists of constant monitoring of the automated processes. This step also uses Process Mining techniques in order to provide insights about process changes.

The case study described by [24] discusses about Purchase-to-Pay (P2P) process consisting of 395315 purchase orders. Two P2P processes were compared based on the performance rate using Celonis ${ }^{4}$. According to his co-founder, Alexandre Rinke, Process Mining provides a much more controlled approach to RPA [28]. The standardized process, consisting of a sequential flow and few deviations obtained a rate of $99,3 \%$, while the complex and non-standardized process got a rate of $55,6 \%$.

Finally, the authors propose 5 best practices for a successful RPA using Process Mining implementation: the selection of suitable processes, process standardization, activities ranking based importance, foundation of a team organizing and monitoring the RPA implementation and continuous monitoring of automated processes.

\footnotetext{
${ }^{4}$ https://www.celonis.com/intelligent-businesscloud/the-cloud-platform/
} 
This paper also talks about a case study of Vodafone. By the instrumentality of Process Mining, Vodafone has been notified about the orders with excessive throughput time. Using RPA, the company processed the orders faster and without errors by achieving an order rate of $92 \%$.

The case study highlights the importance of business processes transparency in order to facilitate the selection of mature processes for automation.

\subsection{Case studies presented by leaders of RPA providers}

Last year, Forrester identified UiPath, Automation Anywhere, and Blue Prism as leaders providing RPA solutions [7]. Blue Prism is also mentioned in the RPA case studies described earlier. This section introduces three cases studies where companies are using RPA solutions provided by top 3 leaders in the domain. These case studies can be found on RPA providers' presentation websites.

\subsubsection{HR at Coca-Cola}

Coca-Cola, world's biggest beverage company, also decided to implement RPA using Blue Prism [29]. They started automate HR audit processes. Processes' selection was made based on crieria like process volume, number of process instances and number of employees involved. After this analysis was performed, 150 processes have been identified to be automatable. Process selection is based on criteria like process volumes, viability or risk level number of people involved.

In HR audit process 8 data sets were used. The process itself is very time consuming as the resulted reports must conform to specific formats. The automated generation of reports using RPA reduced time and offered the employees more time to focus on other tasks. The productivity also increased as robots work 24hours per day instead of 8-hours.

Although Coca-Cola's aim was not to replace employees with robots, the company is planning a staff re-skilling training as HR employees having technical orientation may be also integrated in RPA projects. Furthermore, customer experience improved due to a higher human interaction.

\subsubsection{Customer Identification process in Banking}

Federal Bank is a leading private sector bank headquartered in Kerala, India. The regulations asked for unique customer identification codes for customer management. UiPath assisted the bank to complete this assignment [30].

The results were promising as robots could merge around 250 records in one hour, while employees need a full day. Currently, 15 processes are automated, but the future developments involve 53 processes. Thus, UiPath helped to automate the process by saving time, costs and efforts complying the deadline.

\subsubsection{Accounting processes in Public Sector} Accounting workflows from Australian Post, which has more 4000 post offices countrywide, are optimized using RPA bots [31]. The institution has a huge number of back-offices processes and wanted to automate repetitive tasks. Thus, Automation Anywhere ${ }^{5}$ proposed a free trial and due to the real success of the pilot project, their collaboration continued.

Until now 25 processes are automated. This work is bolster by 120 bots and saves 18000 hours annually, while the costs decreased by $15 \%$.

\section{Discussion}

The table below summarizes the results described in the previous section. Automated processes belonging to case studies of successful RPA implementation are depicted. A detailed discussion and analysis follows next. All companies mentioned in the case studies automated back-office tasks/processes. Frontoffice process automation was only considered within one case study together with backoffice process automation within payment receipt generation process.

\footnotetext{
${ }^{5} \mathrm{https}: / /$ www.automationanywhere.com
} 
Two case studies belonging to outsourcing companies do not mention the tools or methods used in RPA implementation. Blue Prism is mentioned in 3 out of 10 case studies, while Celonis is used in 2 case studies. One case study using UiPath and one using Automation Anywhere are also investigated. An RPA implementation using Deep Convolutional Neural Networks is also introduced.

Table 1. RPA case studies

\begin{tabular}{|l|l|l|l|}
\hline Automated processes & $\begin{array}{l}\text { Back-office } \\
\text { process auto- } \\
\text { mation }\end{array}$ & $\begin{array}{l}\text { Front-office } \\
\text { process auto- } \\
\text { mation }\end{array}$ & $\begin{array}{l}\text { Tool / Method } \\
\text { used }\end{array}$ \\
\hline $\begin{array}{l}\text { SIM swap process and } \\
\text { application to a pre- } \\
\text { calculated credit pro- } \\
\text { cess [18] }\end{array}$ & Yes & Blue Prism \\
\hline $\begin{array}{l}\text { Insurance processes } \\
\text { [25] }\end{array}$ & Yes & No & Blue Prism \\
\hline $\begin{array}{l}\text { Financial processes } \\
{[11],[30]}\end{array}$ & Yes & No & NA \\
\cline { 2 - 4 } $\begin{array}{l}\text { Generation of a pay- } \\
\text { ment receipt [12] }\end{array}$ & Yes & No & UiPath \\
\hline $\begin{array}{l}\text { Automation of data } \\
\text { extraction and pro- } \\
\text { cessing from docu- } \\
\text { ments [27] }\end{array}$ & Yes & No & NA \\
\hline $\begin{array}{l}\text { Purchase-to-Pay } \text { [24] } \\
\text { tional Nop Noural } \\
\text { Yes }\end{array}$ & No & Cetworks \\
\hline $\begin{array}{l}\text { HR audit and Finance } \\
\text { shared services pro- } \\
\text { cesses [29] }\end{array}$ & Yes & No & Blue Prism \\
\hline $\begin{array}{l}\text { Accounting processes } \\
\text { [31] }\end{array}$ & Yes & No & $\begin{array}{l}\text { Automation } \\
\text { Anywhere }\end{array}$ \\
\hline
\end{tabular}

The quantitative analysis is not incorporated within all cases studies mentioned in this paper. The greatest number of automated processes is 25 [31]. Telefónica $\mathrm{O} 2$ [18] used 160 robots performing around 500000 transactions each month within the automation procedure of 15 core processes. The insurance case study presented by Xchanging [25] automated 14 processes by using 27 robots. Coca-Cola [29] identified 150 processes suitable for automation, while Australian Post [31] automated 25 processes by means of 120 robots.

All cases studies confirm that prior RPA project implementation, process assessment is critical.

For example, OpusCapita [11] proposes a lifecycle of RPA projects that starts with a workshop, then the process is evaluated. If the business case is approved, robots configuration starts, while [24] suggests three simple steps: assess, develop and sustain.

Reduced executing time of processes are also mentioned within the investigated case studies. For example, the robots implemented in banking sector [30] could unify 250 records per hour, while an employee needs a full day to execute the same task. The case study of Aguirre and Rodriquez [12] shows a doubling number of executed cases when using RPA, although the mean case duration decreased only by 9 seconds. RPA also helps on costs reduction. For example, Australian Post [31]

\footnotetext{
${ }^{6}$ Two case studies are described in Section 5.1.5.
} 
reports savings of $15 \%$.

In order to select which process/task to be automated, there are some common criteria identified in all case studies: a) high volume of tasks, b) rule-based tasks, c) low complexity tasks/process, d) standardized process, and e) mature process. Successful implementation of RPA also requires an RPA expert [11], [24], [25].

\section{Conclusions}

Robotic Process Automation provides considerable potential in several domains. This paper presented RPA implementation case studies from different domains like telecommunications, insurance, finance, banking, public sector, production of soft drinks and public administration. Although there are traditional systems that support automation, RPA operates and communicates with other applications using front-end level. Due to this reason, the performance is affected and RPA is considered inferior to traditional back-end system automation [11]. Although, RPA represents the leading front-end solution in digital transformation [32].

Companies need to constantly monitor their own business processes in order to identify and optimize processes suitable for RPA because not all processes support automation. High volume of tasks, rule-based tasks or low-complexity tasks are suitable for automation. Moreover, standardized and mature processes are also optimal candidates for automation. Business processes transparency facilitates the selection of mature processes for automation [24].

Another advantage given by RPA refers to cost savings. The studies show that robots implemented in banking sector [30] in customer identification process could examine and merge 250 records each hour, while an employee needs a full day for the same number of records. Also, Australian Post [31] records savings of $15 \%$ since using RPA.

A higher number of executed traces is another advantage brought by RPA. The automation of back-end and front-end processes presented in [12] indicates a doubling number of executed cases when using RPA.

Because employees may be sceptical about RPA, it is important that companies engage them in more interesting and cognitive tasks [17]. The use of Process Mining together with RPA accelerates the implementation of RPA [24].

\section{References}

[1] B. Bygstad, Bendik, "The Coming of Lightweight IT" in Proc. of 23rd European Conference on Information Systems (ECIS), Münster, Germany, 2015, Paper 22.

[2] L.P. Willcocks, M. Lacity and A. Craig, "The IT function and robotic process automation", The Outsourcing Unit Working Research Paper Series (15/05), The London School of Economics and Political Science, London, UK, 2015, pp. 1-39.

[3] E. Penttinen, H. Kasslin, and A. Asatiani, "How to choose between robotic process auto-mation and back-end system automation?" In Proc. of 26th European Conference on Information Systems (ECIS), Portsmouth, United Kingdom, 2018.

[4] W.Huang, Y. Chen, and J. Hee, "STP technology: An overview and a conceptual frame-work" in Information \& Management, vol. 43, no. 3, pp.263-270, 2006.

[5] H.P. Fung, "Criteria, Use Cases and Effects of Information Technology Process Automation (ITPA)" in Advances in Robotics \& Automation, vol. 3, no. 124, 2014.

[6] C. Lamberton, "Get ready for robots -Why planning makes the difference between success and disappointment", EYGM Limited, United Kingdom, 2016.

[7] C. Le Clair, "The Forrester Wave ${ }^{\mathrm{TM}}$ : Robotic Process Automation", Q2 2018.

[8] J. Hindle, M. Lacity, L. Willcocks, and S. Khan, "Robotic Process Automation: Benchmarking the Client Experience. Knowledge Capital Partners", https://static1.squarespace.com/static/58e ceda617bffc $97 \mathrm{~d} 03 \mathrm{~b} 69 \mathrm{da} / \mathrm{t} / 5 \mathrm{~b} 62 \mathrm{bdeaf} 950$ b7f18d967216/1533197807521/RPA++Benchmarking+the+Client+Experience.pdf, 2018, [Accessed: July 12, 2019].

[9] European Patent Office, "European Patent Application Robotic Process Automation - 
EP3112965A1",

https://pa-

tents.google.com/pa-

tent/EP3112965A1/en, 2015, [Accessed:

July 12, 2019].

[10] Gartner, Robotic Process Automation (RPA), IT Glossary, https://www.gartner.com/it-glossary/robotic-process-automation-rpa, [Accessed: July 12, 2019].

[11] A. Asatiani and E. Penttinen, "Turning robotic process automation into commercial success-Case OpusCapita" Journal of Information Technology Teaching Cases, vol. 6 , no. 2, pp. 67-74, 2016.

[12] S. Aguirre and A. Rodriguez, "Automation of a business process using robotic process automation (rpa): A case study" in Workshop on Engineering Applications, pp. 65-71. Springer, Cham, 2017.

[13] K.C. Moffitt, A.M. Rozario and M.A. Vasarhelyi, "Robotic process automation for auditing", Journal of Emerging Technologies in Accounting, vol. 15, no. 1 , pp.1-10, 2018.

[14] Laserfiche, "What is Robotic Process Automation?", https://www.laserfiche.com/ecmblog/what-is-roboticprocess-automation-rpa/\#, [Accessed: July 14, 2019].

[15] P. Harmon, "Harmon on BPM: Robotic Process Automation Comes of Age", https://www.bptrends.com/harmon-onbpm-robotic-process-automation-comesof-age/, 2017, [Accessed: July 14, 2019].

[16] N. Prangnell and D. Wright, "The robots are coming, A Deloitte Insight Report". http://www2.deloitte.com/content/dam/Deloitte/uk/Documents/finance/deloitte-uk-financerobots-are-coming.pdf, 2015, [Accessed: July 20, 2019].

[17] M. Lacity and L. Willcocks, "What Knowledge Workers Stand to Gain from Automation" Harvard Business Review, https://hbr.org/2015/06/what-knowledgeworkers-stand-to-gain-from-automation, 2015, [Accessed: July 20, 2019].

[18] M. Lacity, L.P. Willcocks and A. Craig, "Robotic process automation at Telefónica O2" The Outsourcing Unit Working Research Paper Series (15/02), The London
School of Economics and Political Science, London, UK, 2015, pp. 1-19.

[19] M. Weske, W.M. van der Aalst, and H.M.W. Verbeek, "Advances in business process management", in Data \& Knowledge Engineering, vol. 50, no. 1, pp.1-8, 2004.

[20] M. Lacity and L. Willcocks, "Robotic Process Automation: The Next Transformation Lever for Shared Services", The Outsourcing Unit Working Research $\mathrm{Pa}$ per Series (16/01), The London School of Economics and Political Science, London, UK, 2016, pp. 1-39.

[21] S. Mohapatra, "BPR and Automation", In: Business Process Reengineering, Management for Professionals, Springer, Boston, MA, 2013.

[22] L. Willcocks, M. Lacity, "Service Automation: Robots and the Future of Work" Steeve Brokes Publishing, Warwickshire, 2016.

[23] W.M.P. van der Aalst, "Process Mining Data Science in Action" 2nd edition, Springer, Heidelberg, 2016.

[24] J. Geyer-Klingeberg, J. Nakladal, F. Baldauf and F. Veit, "Process Mining and Robotic Process Automation: A Perfect Match", in Proceedings of the Dissertation Award, Demonstration, and Industrial Track at BPM 2018, pp. 9-14.

[25] L.P. Willcocks, M. Lacity and A. Craig, "Robotic Process Automation at Xchanging", The Outsourcing Unit Working Research Paper Series (15/03), The London School of Economics and Political Science, London, UK, 2015, pp. 1-26.

[26] P. Hallikainen, R. Bekkhus, and S.L. Pan, "How OpusCapita Used Internal RPA Capabilities to Offer Services to Clients" in MIS Quarterly Executive, vol. 17, no. 1, 2018.

[27] C. Houy, M. Hamberg and P. Fettke, "Robotic Process Automation in Public Ad-ministrations" Digitalisierung von Staat und Verwaltung, Lecture Notes in Informatics (LNI), Gesellschaft für Informatik, pp. 67-74, 2019. 
[28] S. Lazarus, "Achieving a Successful Robotic Process Automation Implementation: A Case Study of Vodafone and Celonis", https://spendmatters.com/2018/06/07/achieving-asuccessful-robotic-process-automation-implementation-a-case-study-of-vodafone-andcelonis/, 2018, [Accessed: July 22, 2019].

[29] Blue Prism, "Coca-Cola Extends Business Services Capacity and Improves Performance with RPA", https://www.blueprism.com/resources/case-studies/cocacola-extendes-business-services-capacityand-improves-performance-with-rpa, May 2018, [Accessed: August 15, 2019].

[30] UiPath, "Federal Bank Partners with UiPath to Meet a Nearly Impossible Compli- ance Deadline", https://www.uipath.com/solutions/customer-success-stories/federal-bank, [Accessed: August 15, 2019].

[31] Automation Anywhere, "Australia Post Optimizes Accounting Workflows with RPA Bots", https://www.automationanywhere.com/casestudy-australia, May 2019, [Accessed: August 15, 2019].

[32] D. Mills, "Why RPA can be the Backbone for Front End Enterprise Digital Transformation", https://www.jacada.com/blog/why-rpa-can-be-the-backbone-for-front-end-enterprise-digitaltransformation, [Accessed: August 15, 2019].

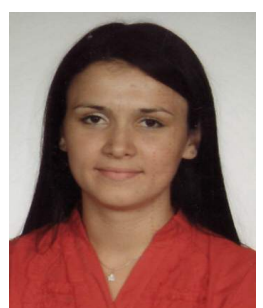

Cristina-Claudia OSMAN has graduated the Faculty of Economics and Business Administration, Babeş-Bolyai University, Cluj-Napoca in 2008. She holds a bachelor degree in Business Informatics, master degree in E-Business and a $\mathrm{PhD}$ degree in Cybernetics and Economical Statistics from 2014. Her current research interest includes Process Mining, Business Process Management, Business Process Modelling and Text Mining. 